\title{
Unicompartmental Knee Arthroplasty for Severe Osteoarthritis and Pseudarthrosis in a Patient with Neurofibromatosis
}

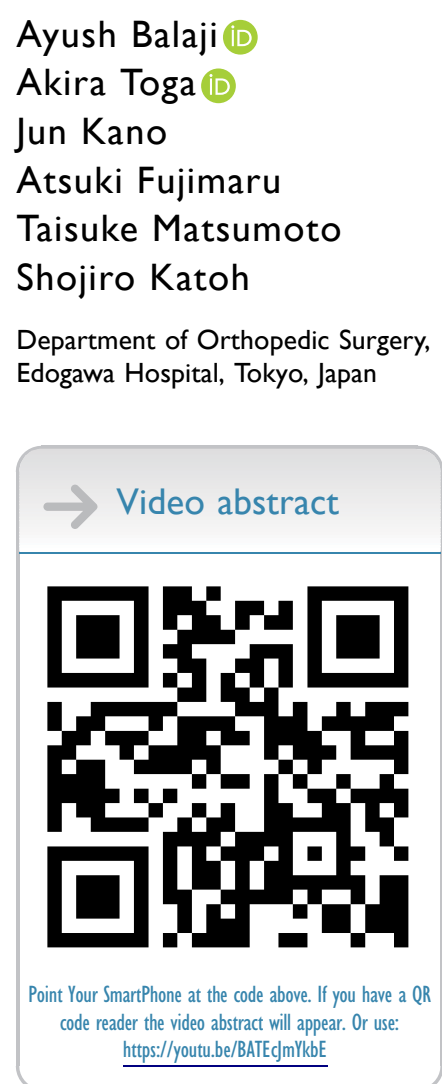

Correspondence: Akira Toga Department of Orthopedic Surgery, Edogawa Hospital, 2-24- 8 Higashikoiwa, Edogawa-ku, Tokyo, 160-8582, Japan

Tel +8I-3-3673-I22I

Fax +8I-3-3673-I229

Email akira.toga@gmail.com

\begin{abstract}
We describe the case of a 76-year-old Asian female patient who presented with severe pain and a valgus deformity of the right knee. Her past medical history included neurofibromatosis, which resulted in a severe anterior slope of the right knee, limb shortening, and congenital pseudarthrosis. She was diagnosed with severe anterolateral osteoarthritis and eburnation of the right knee that was treated surgically with a lateral unicompartmental knee arthroplasty (UKA). Bone and cartilage fragments were removed from the joint space and a UKA implant $\left(\right.$ Zimmer $^{(B)}$ ) with dimensions of $29 \mathrm{~mm} \times 50 \mathrm{~mm}$ was inserted. Perioperative imaging revealed that the procedure resulted in the correction of the valgus deformity. Pain was reduced and the patient was able to walk for 10 meters with support during the immediate postoperative period. One week post-surgery, the patient sustained an oblique tibial fracture that extended from the medial edge of the implant to the medial slope of the proximal tibia. This complication may have been attributed to large implant size or sagittal overcutting. The fracture was treated surgically with a rotated anterolateral locking plate (A.L.P.S ${ }^{\circledR}$ ) inserted into the distal tibia. The patient was capable of ambulation at full weight load at two months after the second procedure. It is critical to recognize that there are no standard protocols that can be used to guide the treatment of neurofibromatosis-induced osteoarthritis. The specific preoperative condition of the individual patient plays a large role in determining the appropriate treatment option. In this case, the availability of custom-fitted UKA implants might have improved outlook, we understand that these devices are costly and may not be available at all hospitals. However, we strongly believe that the "gold standard" in these cases is patient-specific treatment that addresses the issues of the highest concern using the resources that are available at that time.
\end{abstract}

Keywords: skeletal deformities, limb shortening, stress fracture, knee implants

\section{Introduction}

In this report, we review the case of a 76-year-old Asian female with a past medical history of neurofibromatosis (NF), congenital pseudoarthrosis, and dysregulated osteogenesis who presented with osteoarthritis (OA) of the right knee. NF is a genetic disorder that results in tumor growth throughout the nervous system. ${ }^{1}$ Type 1 NF is caused by mutations in a gene on chromosome 17 that encodes neurofibromin, a GTPase-activating protein that regulates cell growth. Type 1 NF is a rare disorder with a reported incidence of 1 in 4000 live births worldwide. ${ }^{2}$ Skeletal deformities are observed frequently among patients diagnosed with NF. A review of the medical literature suggested that NF is associated with a higher prevalence of 
skeletal deformities, including congenital pseudoarthrosis than was previously recognized. ${ }^{3}$ Arthritis has also been diagnosed in patients with NF, although all but one report to date have focused on pathology at the hip joint. ${ }^{4}$ There are no reports detailing outcomes of knee arthroplasty and open reduction internal fixation procedures, in patients with NF. As such, other than amputation, no comprehensive treatment plans have been established to guide the management of orthopedic manifestations of NF at the knee joint.

\section{Case Report}

A 76-year-old woman presented at our outpatient clinic with symptomatic OA of the right knee. At admission her height was measured to be $139.8 \mathrm{~cm}$ and her weight was measured to be $46.5 \mathrm{~kg}$. Her past medical history included $\mathrm{NF}$, congenital pseudarthrosis, and combined deformities of the tibia and fibula that resulted in the shortening of the right lower limb. At presentation, her examination revealed a limited active range of motion at the right knee joint (from 0 to $90^{\circ}$ ), a valgus deformity of $14^{\circ}$ using the femoro-tibial angle measurement, severe pain, and a progressively worsening antalgic gait due to the preexisting limb shortening. These deformities, including pseudoarthrosis, are consistent with skeletal deformities previously described in patients diagnosed with NF. ${ }^{5}$ She was diagnosed with Grade $4 \mathrm{OA}$ as per the KellgrenLawrence (KL) classification based on the results of radiographic imaging and the physical examination (Figure 1). Her walking distance was limited due to pain. Passive range of motion studies revealed significant hyperextension $\left(-10^{\circ}\right)$ consistent with an increased risk for dislocation. The physical exam also revealed peroneal nerve palsy and café au-lait spots across her entire body, the latter finding indicative of advanced NF. She had previously undergone a bone transplant at the fibula with a graft taken from the tibia approximately 50 years prior; as such, there was only a limited amount of viable bone remaining to support the successful implantation of a prosthesis. As there was not sufficient viable bone stock available to support the ready-made intramedullary stem used in total knee arthroplasty (TKA; Figure 2), our initial plan was to perform a bicompartmental knee arthroplasty (BKA). Furthermore, as part of the preoperative procedure at our hospital, we conducted a bone density test. This evaluation revealed a femoral bone density of $0.525 \mathrm{~g} / \mathrm{cm}^{3}$, which affirmed our decision to avoid a TKA,
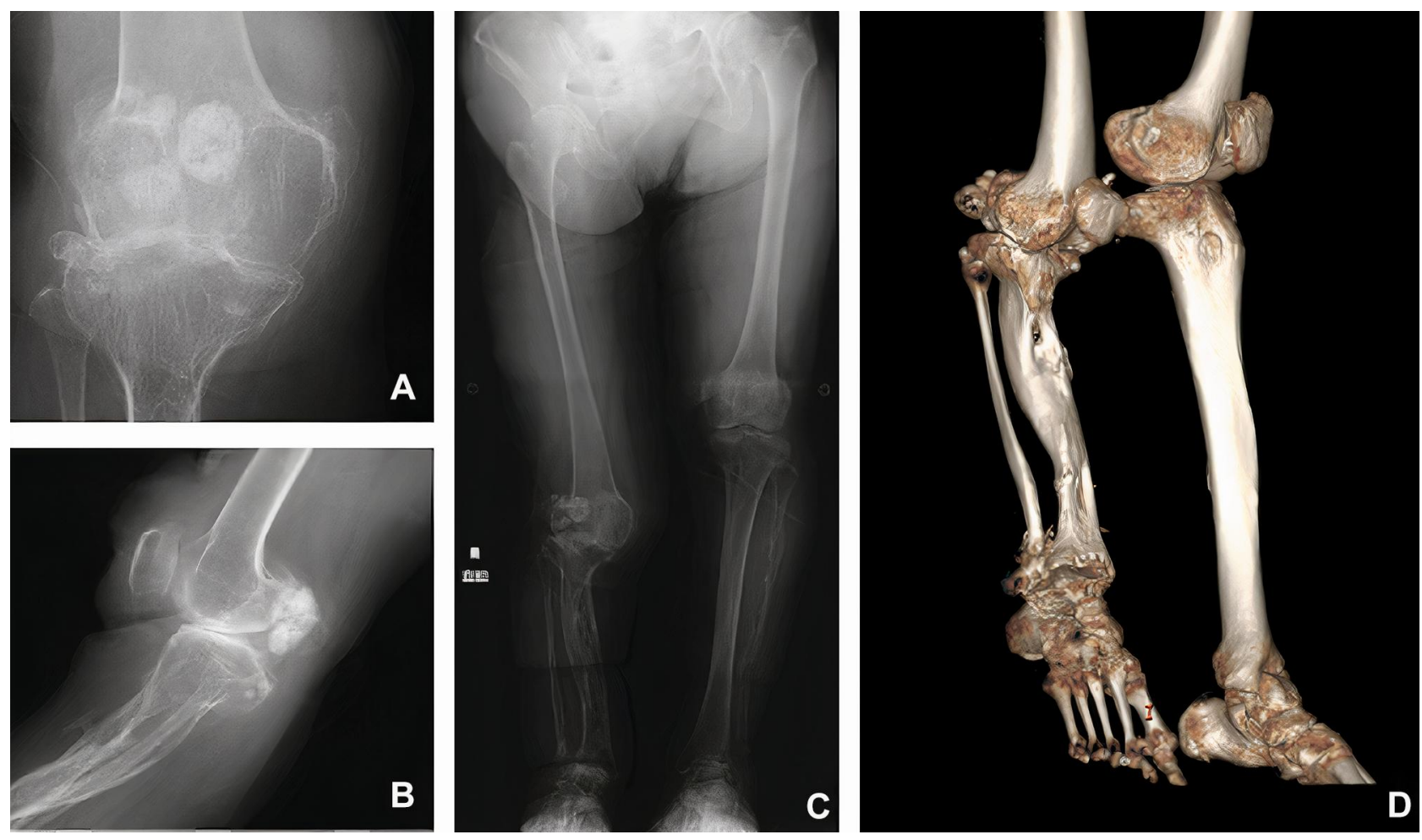

Figure I (A) Radiography of the right leg documenting severe bone-on-bone contact and bone bruising. (B) Lateral radiograph of the right knee documenting hyperextension with a severe anterior slope. (C) Anteroposterior radiograph documenting the extent of limb shortening. (D) Three-dimensional computed tomography (CT) scan documenting the tibial deformity and osteoarthritis. 

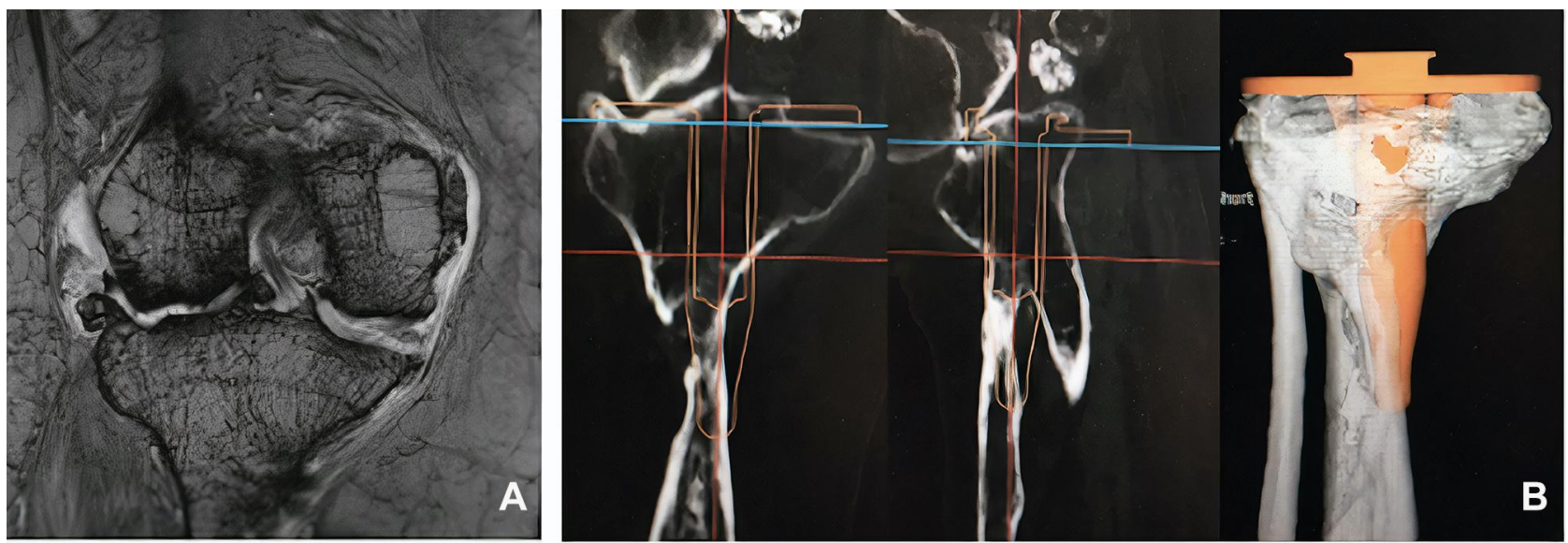

Figure 2 (A) T2-weighted coronal MRI depicting soft tissue at the right knee and indicating isolated osteoarthritis on the lateral side. (B) TKA implant templating process revealed inadequate viable bone stock that would be unable to support an implant.

as the quality of the available bone stock would unlikely be sufficient to support adequate fixation of the larger implants required in this procedure. Magnetic Resonance Imaging (MRI) was performed to provide maximum visualization of the lesion, given the discrepancies in the anatomical structures and also to evaluate the soft tissue condition (Figure 2). Although a bicompartmental knee implant was templated, the cartilage on the medial side of the joint was found to be intact on the coronal MRI scan, thereby presenting a contraindication to this procedure (Figure 2). Additional radiographic studies revealed that the patient had both femoral and tibial insufficiency and a significant reduction in the bone stock available for the positioning of an implant that required a tibial stem. As this patient also exhibited problems with osteogenesis due to NF, all procedures were planned with the goal of limiting the degree of tissue damage. With this in mind, we considered unicompartmental knee arthroplasty (UKA) as a minimally invasive procedure that might be used for the successful amelioration of her OA symptoms. A lateral UKA to fix the valgus deformity was discussed with the patient as a means to provide adequate relief from the pain resulting from the bone-on-bone contact at the lateral side as well as to address the problem of hyperextension. The patient agreed to this course of action. Preoperative planning focused on addressing the issues associated with bone-on-bone contact as well as repair of the valgus deformity with the goal of promoting stability at the knee. The range of motion was verified preoperatively, and femoral and tibial contact points were mapped across the full range of motion to determine the degree of the anterior slope. Unfortunately, the dimensions of the anterior slope precluded the use of an implant of the size that would be desirable given the patient's tibial dimensions. We were also aware of the fact that the risks of dislocation are increased in NF patients due to characteristic joint hypermobility and problems with soft tissue in areas such as the skin, menisci, and musculature. ${ }^{6}$

During surgery, the surrounding tissue was debrided, and a large fragment of debris was removed from the joint space. Visual inspection of the knee confirmed the diagnosis of isolated lateral $\mathrm{OA}$ with intact medial articulation (Figure 3). A femoral unicompartmental knee system (Physica ZUK; size D) implant and a $29 \mathrm{~mm} \times 50 \mathrm{~mm}$ tibial implant $\left(Z_{i m m e r}{ }^{\mathbb{B}}\right)$ were inserted. Perioperative radiographs revealed that, although the trial implant was slightly larger than ideal, synchronous soft tissue balance of the knee was confirmed (Figure 4). Unfortunately, as customized implants were not available, space remained between the femoral implant and the femoral condyle.

Prior to final fixation, the articulation site was copiously irrigated to facilitate the penetration of cement into the femur and tibia. The final implant was then inserted into small holes that were drilled into the tibia and femur that also facilitated cement adhesion. The cement was molded to fill the gap observed during the trial implantation (Figure 3).

Postoperative imaging and physical examination revealed that the anterior tibial slope was repaired and that the valgus deformity was reduced to $5^{\circ}$ from the $14^{\circ}$ determined preoperatively (Figure 5). The patient was capable of ambulation within 24 hours after completion of the procedure. However, the patient developed a varus thrust and continued to require epidural analgesia for 48 

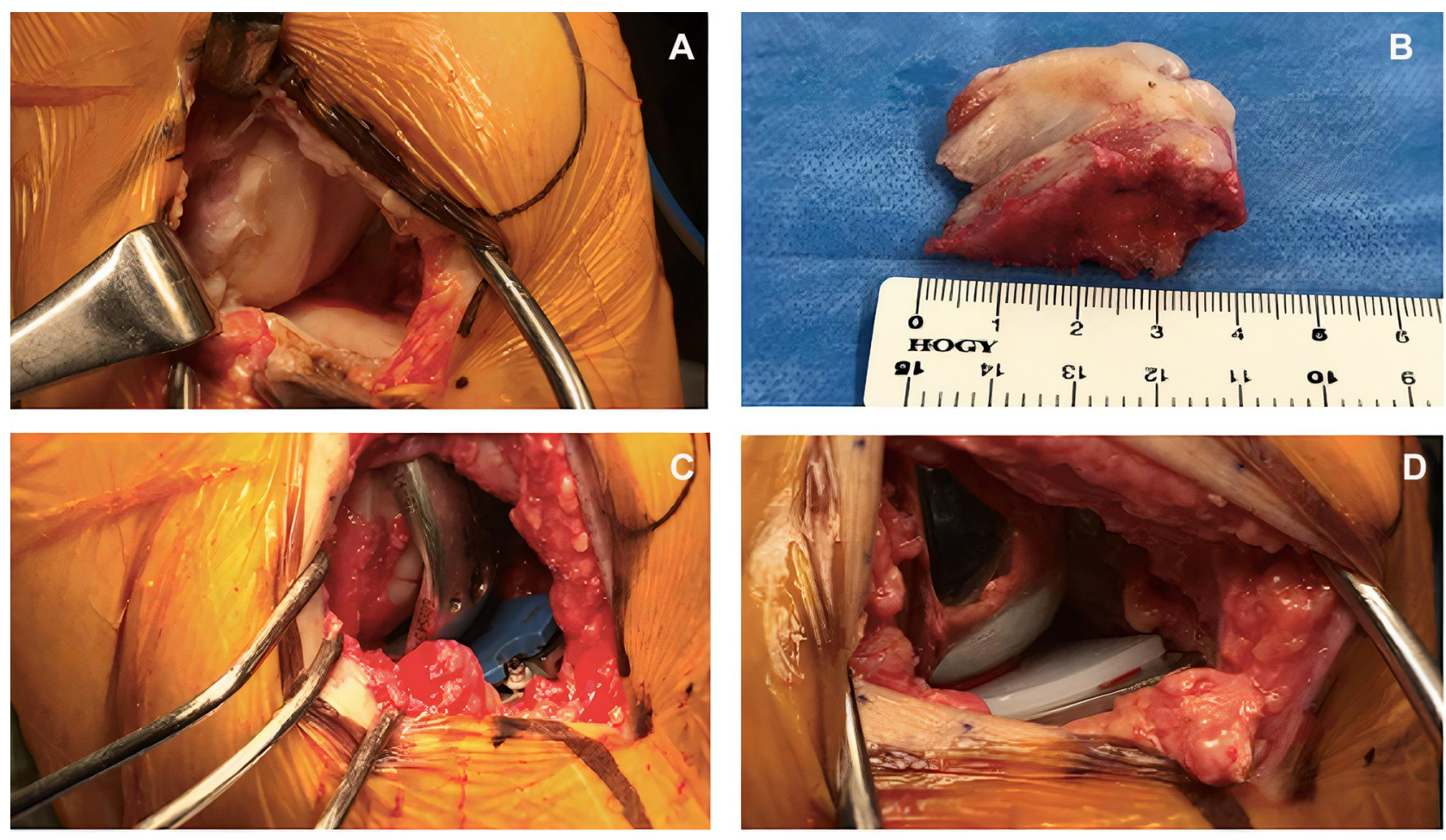

Figure 3 (A) Perioperative photographs documenting the extent of cartilage damage at the lateral aspect of the right knee. (B) Excised portion of the proximal tibia with dimensions approximately $40 \times 30 \times 20 \mathrm{~mm}$. (C) Placement of the trial implant with the smallest available polyethylene spacer insert. (D) Final UKA implant with cement and polyethylene insert as shown.
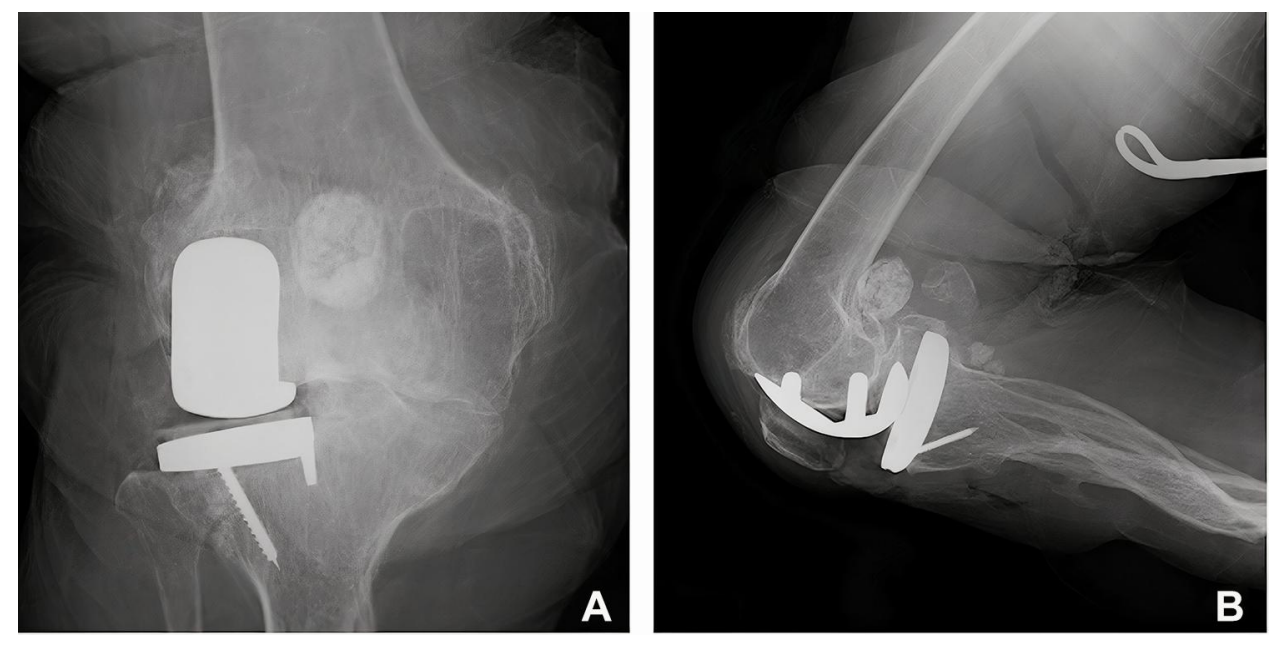

Figure 4 Perioperative radiographs of the trial implant taken from the anteroposterior direction during $(\mathbf{A})$ extension and from the lateral direction during $(\mathbf{B})$ flexion; note the visible gap between the femoral condyle and implant in the latter image.

hours after the procedure. The rehabilitation plan included assisted range of motion and partial weightbearing walking exercises. The patient exhibited a myopathic gait; this was deemed acceptable given her preexisting musculoskeletal condition. One week after the procedure, the patient continued to ambulate with the assistance of two walking poles and a knee brace.
She reported that soreness developed after walking for approximately 20 meters; this was attributed to preoperative muscle stiffness (Video 1). The active range of motion extended from 0 to $110^{\circ}$; this represented a significant improvement over her preoperative range of 0 to $90^{\circ}$. Ankle movement was restricted due to preexisting peroneal nerve palsy and mild pain was present 

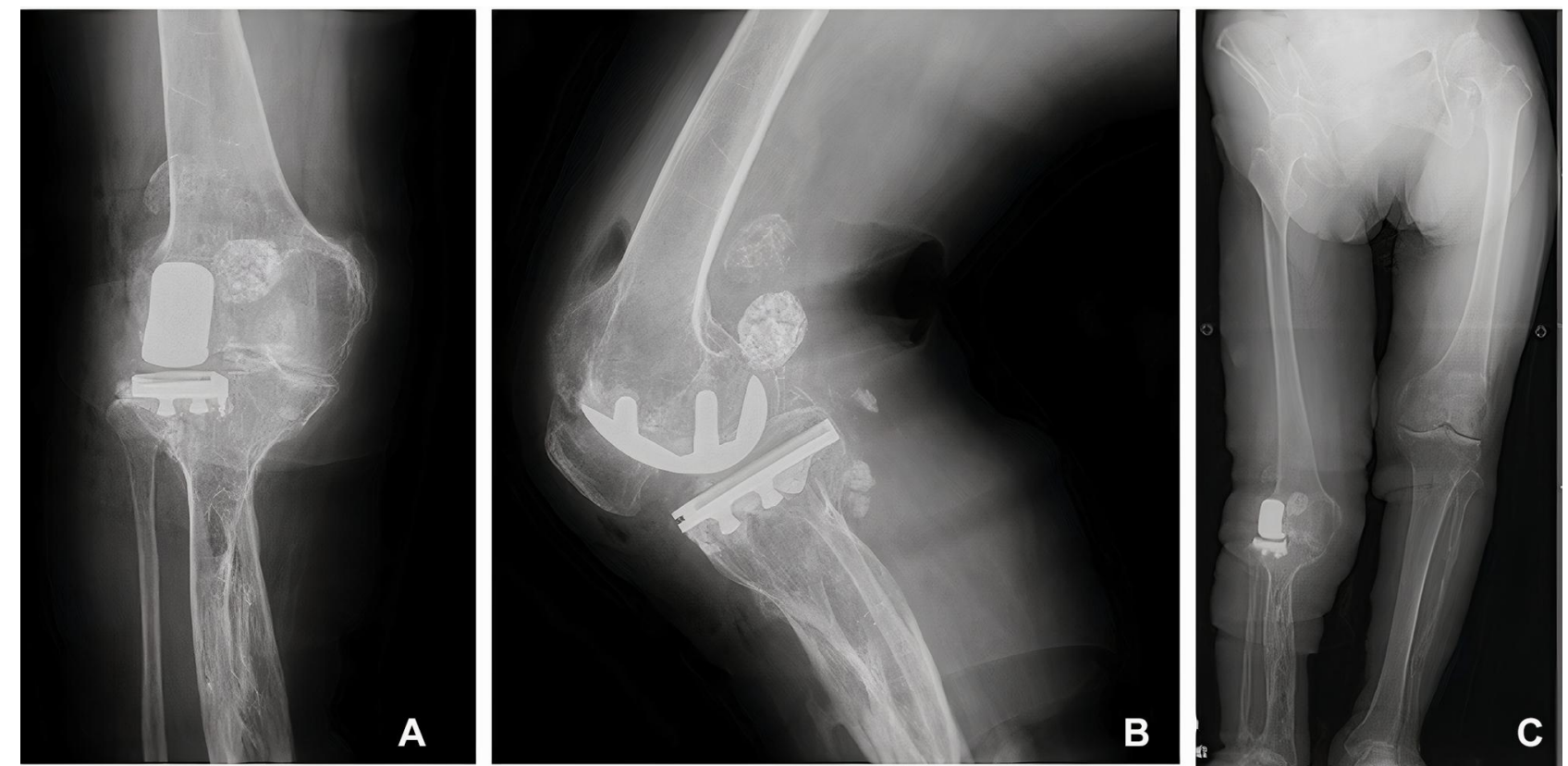

Figure 5 (A) Anteroposterior and (B) lateral postoperative radiographs of the final UKA implant, documenting correction of the anterior slope. AP radiograph showing that the valgus deformity was resolved postoperation $(\mathbf{C})$.

after the surgery. The most substantial functional gains from the lateral UKA procedure were the improvements in the knee alignment and the reduction in the valgus deformity. Both of these corrections contributed to significant improvements in patient ambulation.

Two weeks after the procedure, the patient experienced a fall that resulted in a stress fracture of the tibial plateau. The lesion was diagnosed as a proximal oblique fracture of the tibia. This was most likely due to a mismatch between the implant and patient-specific mechano-physiology which may have potentially resulted in sagittal overcutting. The tibial UKA component was not displaced (Figure 6). Three days post-fracture, a surgical procedure involving a locking plate was planned. A distal tibia anterolateral plate (A.L.P.S ${ }^{\circledR}$ ) with nine holes was rotated upside down to achieve successful fixation of the fracture. Eight locking cortical screws were inserted; postoperative imaging documented adequate fixation and support (Figure 6).
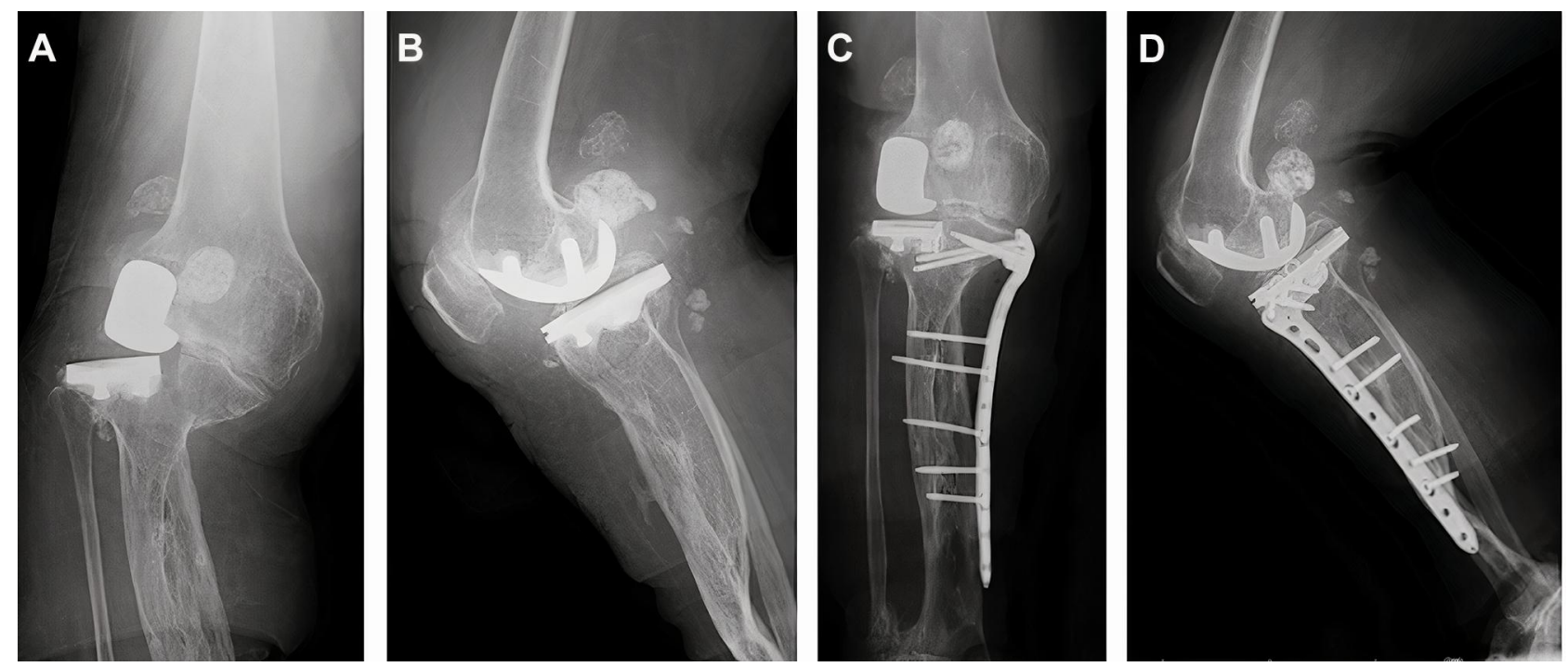

Figure 6 Postoperative (A) anteroposterior and (B) lateral tibial plateau lesion diagnosed as an oblique fracture of the proximal tibia. (C) Anteroposterior postoperative radiograph of the distal tibia locking plate indicating adequate fixation. (D) Lateral postoperative radiograph of the distal tibia locking plate indicating adequate fixation. 
Given the high risk for dislocations, continuous passive motion therapy was contraindicated. Within five days after the second procedure, the patient reported only minimal pain and was able to undergo successful rehabilitation to improve her range of motion. We note that her participation in the standard rehabilitation program was interrupted due to the ongoing impact of the COVID-19 pandemic. This was unfortunate, as adequate postoperative rehabilitation can be critical for recovery. The patient was provided with a fixed knee brace to stabilize her leg during the first two months after the insertion of the locking plate. As the COVID-19 pandemic was then under adequate control in the region, the patient was able to follow a rehabilitation plan that included both passive and active progressive range of motion exercises, partial weight-bearing walking regimens, and measures designed to manage pain and swelling. The patient was put on a partial weight-bearing walking regimen $(30 \%$ to start, gradually increased to $100 \%$ body weight every week) beginning at one week postoperatively. At the two-month postoperative checkup, the patient was able to walk with a full weight load without assistance (Figure 7). Four months after the procedure, the patient reported significant improvements with respect to pain and active range of motion. The patient returned to her activities of daily life and did not need a knee brace or supporter (Video 2). Radiographic images revealed callus formation at the fracture site, indicative of new bone formation. We planned to follow this patient on a more long- term basis to manage pain and swelling and to maintain the improved range of motion and weight-bearing capability. However, the overall impact of COVID-19 limited the extent to which we could follow this patient after the fivemonth check-up. Nonetheless, the patient planned to continue with the prescribed rehabilitation plan. The patient presented for a 7-month postoperative checkup and reported some residual pain that she managed effectively with the pain medication regimen that was prescribed. She reported that she was able to walk on most days and carry out day-to-day activities without difficulty. On certain days, she noted some instability at her knee joint which is likely attributable to the postoperative fracture. Further radiographic studies revealed appropriate fracture healing, and her physical exam documented appropriate knee alignment.

Although the patient was capable of ambulation prior to the UKA procedure, she relied on external support and experienced substantial pain due to osteoarthritis and poor knee alignment. The contraindications to a conventional TKA outweighed the possible benefits that might be achieved in this case. A UKA seemed to be a more appropriate solution toward resolving the pain resulting from bone-on-bone contact and the valgus deformity. The UKA procedure is less invasive, maintains the kinematics at the knee, preserves more bone, and facilitates easier revision surgery if this becomes necessary.
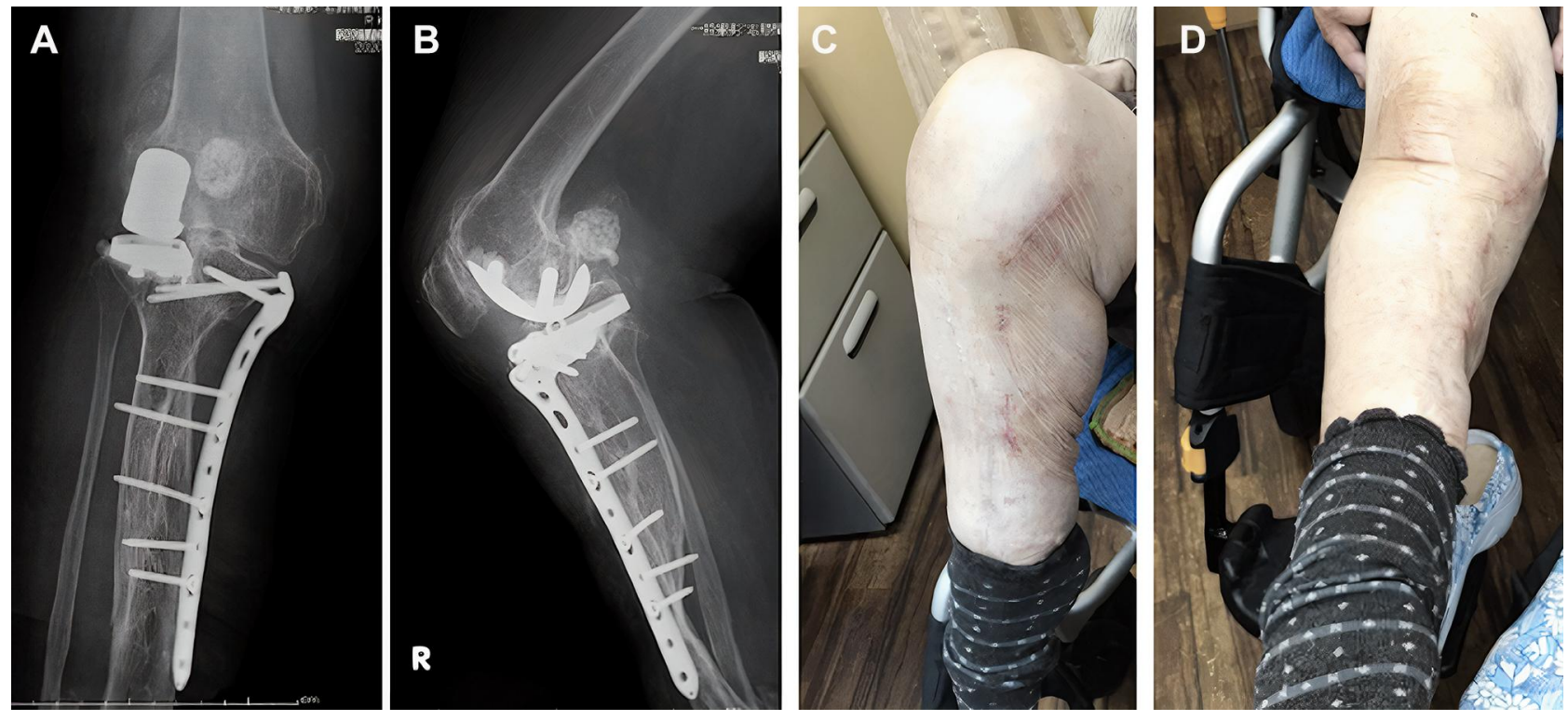

Figure 7 (A) Anteroposterior and (B) Lateral radiographs at five months postoperatively revealing callus formation at the fracture site, indicative of new bone formation. Shown are images of the right knee under (C) flexion and (D) extension. The range of motion improved, and hyperextension was corrected. 
The decision to perform a UKA was controversial due to the unique complexities associated with this patient. However, these therapeutic modalities resulted in a significant improvement in the patient's quality of life. This outcome was evaluated quantitatively by the Knee injury and Osteoarthritis Outcome Score (KOOS) which is included in the Supplemental Files (Table S1). An increase of 8-10 points on the KOOS total score has been clearly established as a clinically significant improvement. Our patient exhibited a 23.2-point increase in KOOS in response to treatment. This finding indicates that the patient experienced significant benefit from the complete treatment plan. Specifically, the patient reported improvements in areas including but not limited to, a reduction in pain, reduced stiffness, improved mobility, and a better quality of life.

\section{Discussion}

UKA is a well-established intervention for isolated OA. As UKA is less invasive than TKA, this method is preferred by both surgeons and their patients. The less invasive approach is typically favored in patients with multiple comorbidities, as it reduces the risk of additional complications and intractable pain. In this patient, pain reduction and avoiding tissue damage were the two most significant factors that led to the choice of UKA over TKA. Although the desired outcome of surgical procedure was achieved (fixing the valgus deformity and anterior slope), this case illustrates the substantial hurdles associated with NF as a fracture occurred postoperatively. Given the increasing prevalence of conditions that promote joint damage, including osteoporosis, pseudoarthrosis, obesity, and limb deformities, the problems associated with ready-made implants have become clear. Most notably, ready-made implants are not suitable for all patients due to variations in both bones and the condition of the adjacent soft tissues. Recent reports indicate that 9-14\% of patients diagnosed with congenital pseudarthrosis may ultimately undergo limb amputation due to complications of their disease together with the inadequacy of currently available treatment options. ${ }^{7,8}$ Our case clearly illustrates the challenging aspects of treating patients with this disorder. This is a larger problem for patients undergoing UKA compared to TKA, as the former procedure involves only minimal disruption of the condylar surfaces and does not involve removal of the anterior cruciate ligament (ACL) or posterior cruciate ligament (PCL). In this case, TKA was not performed due to the ongoing viability of the ACL, the intact medial cartilage, and the unavailability of TKA implants that were appropriate for this patient given the lack of bone stock. During the templating process, we found that the implants protruded from the tibial surface and increased the risk for fracture; this was the case even for those implants with the smallest available stem sizes. As such, UKA was considered to be better suited for this patient. However, even with this modified procedure, the oversized implant contributed to the fracture of the tibial eminence. The gradual degradation of the tibial surface due to the oversized implant caused the surrounding bone to become more susceptible to fracture. A tibial plateau fracture presents the possibility of bone fragmentation and damage to soft tissue.

We addressed this problem with a second procedure that involved the use of locking plates. Results from previous studies suggest that locking plate and screw systems facilitate adequate fixation of the fracture and limit the potential for changes in the position of bone segments. ${ }^{9}$ Our patient exhibited the same range of motion as that recorded before the onset of OA symptoms within 48 hours after the surgery. Furthermore, the tibial deformity resulting from pseudoarthrosis was of large concern when designing a rehabilitation program, as weight-bearing exercises may exert significant amounts of shear stress onto an already degenerated tibia. In this case, while both the ACL and PCL were elongated, the medial collateral ligament and the lateral collateral ligament were both functioning adequately; as such, there was adequate stability of the right knee. We also note that the application of cement was of paramount importance to the success of this procedure because the idiosyncratic characteristics of this patient's knee did not fully match the assumptions made by the implant designers. Cases such as these illustrate the need for accessible custom-fitted implants that can be designed to suit the articular surfaces of individual patients in a more precise fashion. As shown here, the use of unsuitable industry-standard implant sizes may increase the risk of postoperative complications, including ongoing degradation of the surrounding tissue. An ideal device for this patient would be a redesigned version of a hinged TKA implant that could be attached to the tibia with side locking plates rather than a stem. We believe that custom implants are necessary and should be available specifically because of rare cases like this. The purpose of custommade patient-specific implants is to provide a treatment option for rare cases and patients unable to tolerate 
conventional implant technology. We strongly believe that access to a custom-made implant would have improved the postoperative outlook of this patient. It is interesting to consider the fact that these deformities, combined with the risks of postoperative complications, may be among the primary reasons underlying the use of amputation as a primary treatment option for these patients. ${ }^{8}$ Although amputation was considered, our patient had a long history of learning to adjust to her shortened limb; as such, we did not want to perform a drastic procedure that would result in a large shift in her overall balance.

Hyperextension of the knee associated with an increased anterior tibial slope resembles the genu recurvatum deformity; the latter condition is typically addressed with an osteotomy or arthroplasty procedure using rotating hinge implants. ${ }^{10}$ Although rotating hinge implants were available to us, the tibial insufficiency, history of fracture, and low levels of available bone stock suggested that this type of device would not work for this patient. Genu recurvatum deformities are unusual and have been reported in fewer than $1 \%$ of patients undergoing knee arthroplasty. ${ }^{11}$ In our case, these findings led us to choose arthroplasty along with osteotomy as the preferred treatment option as the size and setting of the implant could be used to correct the anterior slope. However, the additional conditions associated with NF added to the more challenging nature of this case. In this specific case, the condition of the surrounding soft tissue facilitated rotation and fixation of the distal tibia plate; we recognize that this may not always be the case. Utmost care should be taken to avoid complications, given that patients with NF may not tolerate treatment with many of the more conventional treatment options. ${ }^{12}$ Surgeons and implant manufacturers need to acknowledge the potential for postoperative fracture due to poorly fitting implants when determining the best course of action for an individual patient. It will also be important to focus on the prioritization of specific aspects of the individual condition. For example, in some cases, one might prioritize the impact of limb shortening over that associated with hyperextension. By contrast, in our case, the priority was to resolve the osteoarthritis and the effects of hyperextension. Therefore, a minimally invasive approach was the preferred treatment option. Taken together, our findings underscore the fact that the orthopedic manifestations of NF pose a significant challenge for orthopedic surgeons. In this case, the complexities associated with the multiple complications of NF necessitated careful planning and specific precautions. Various patient specific treatment options should be designed and evaluated prior to surgery. Although customized implants for rare and highly complex cases may serve to improve patient outlook, we understand that these resources are costly and may not be available at all hospitals. However, we strongly believe that the "gold standard" in these cases is patient-specific treatment. Physicians and surgeons should collaborate with the patient with a focus on addressing the issues of the highest concern that can be treated using the available resources. Although we understand that the procedure that we devised for this case may not be fully generalizable due to the nature and complexities of this specific patient, our experience highlights how the development of unconventional methods using conventional resources can ultimately result in an improved quality of life. In complex cases such as this, effective communication with the patient and discussions with multiple clinicians is of paramount importance, as these steps will help us to predict complications and to determine a strategy to yield the best possible outcome.

\section{Abbreviations}

$\mathrm{NF}$, neurofibromatosis; OA, osteoarthritis; ACL, anterior cruciate ligament; PCL, posterior cruciate ligament; UKA, unicompartmental knee arthroplasty; TKA, total knee arthroplasty; BKA, bicompartmental knee arthroplasty; MRI, magnetic resonance imaging; CT, computed tomography; KOOS, Knee injury and Osteoarthritis Outcome Score.

\section{Informed Consent}

Informed consent was obtained from the patient for publication.

\section{Acknowledgments}

The authors would like to thank Medical Journal Editors (MJE) for assistance in the editing of our manuscript.

\section{Funding}

This study was approved by the review board at Edogawa Hospital. The authors received no financial support for the research, authorship, and/or publication of this article.

\section{Disclosure}

The authors declare no potential conflicts of interest. 


\section{References}

1. Le C, Bedocs PM. Neurofibromatosis. [Updated 2020 Aug 10]. In: StatPearls [Internet]. Treasure Island (FL): StatPearls Publishing; 2021. Available from: https://www.ncbi.nlm.nih.gov/books/ NBK459329/. Accessed May 05, 2021.

2. About Neurofibromatosis | NHGRI [Internet]. Genome.gov. 2016. Available from: https://www.genome.gov/Genetic-Disorders /Neurofibromatosis. Accessed May 05, 2021.

3. Hilde B, Eric L, Johan L, Armand L. Prevalence of neurofibromatosis type 1 in congenital pseudarthrosis of the tibia. Eur $J$ Pediatr 2016;175(9):1193-1198. doi:10.1007/s00431-016-2757-z

4. Alrumaih H, Ilyas I, Kashif S. Neurofibromatosis induced hip arthritis. An unusual presentation. Am J Case Rep. 2014;15:79-81. doi:10. 12659/AJCR.889726

5. Richette P, Claudepierre P, Voisin MC, et al. Hemarthrosis: an unusual complication of type 1 neurofibromatosis. Arthritis Rheum. 2001;44 (9):2134-2137. doi:10.1002/1529-0131(200109)44:9<2134::AIDART366>3.0.CO;2-0
6. Galbraith JG, Butler JS, Harty JA. Recurrent spontaneous hip dislocation in a patient with neurofibromatosis type 1: a case report. $J$ Med Case Rep. 2011;5:106. doi:10.1186/1752-1947-5-106

7. Shah H, Rousset M, Canavese F. Congenital pseudarthrosis of the tibia: management and complications. Indian J Orthop. 2012;46 (6):616-626. doi:10.4103/0019-5413.104184

8. Patel J, Whiting J, Jones D. Secondary knee osteoarthritis due to neurofibromatosis type 1 treated with above the knee amputation: a case report. Case Rep Orthop. 2013;2013:782106. doi:10.1155/ 2013/782106

9. Cronier P, Pietu G, Dujardin C, Bigorre N, Ducellier F, Gerard R. The concept of locking plates. OTSR. 2010;96(4):S17-S36.

10. Chulsomlee K, Kulachote N, Wongsak S, et al. Severe genu recurvatum deformity treated with primary rotating hinge replacement: a report of 2 cases. Thai J Orthop Surg. 2017;41(3 - 4):23-29.

11. Matsuda S. Total knee arthroplasty in patients with genu recurvatum. Orthop Proceed. 2016;98-B(Suppl 3):26.

12. Tangsataporn S, Shakib A, Kuzyk PR, et al. Secondary hip osteoarthritis due to neurofibroma treated with total hip replacement. Case Rep Orthop. 2012;2012:173921. doi:10.1155/2012/173921

\section{Publish your work in this journal}

Orthopedic Research and Reviews is an international, peer-reviewed, open access journal that focusing on the patho-physiology of the musculoskeletal system, trauma, surgery and other corrective interventions to restore mobility and function. Advances in new technologies, materials, techniques and pharmacological agents are particularly welcome. The manuscript management system is completely online and includes a very quick and fair peer-review system, which is all easy to use. Visit http://www.dovepress.com/testimonials.php to read real quotes from published authors. 